\title{
PROGRESSIVE SAR IMAGING TECHNIQUE
}

\author{
Kaizhi Wang ${ }^{1}$, Xingzhao Liu', Member, IEEE, Wenxian Yu', Junli Chen ${ }^{2}$ and Guozhong Chen ${ }^{2}$ \\ ${ }^{1}$ EE Department of Shanghai Jiaotong University \\ ${ }^{2}$ Shanghai Institute of Satellite Engineering
}

\begin{abstract}
A progressive SAR imaging technique is proposed as a novel SAR raw data processing scheme in this paper. Different from the classic SAR imaging algorithms which focus the SAR raw data as a whole despite of targets or clutters, the novel technique focuses the SAR raw data pixel by pixel or in a group of pixels via Atomic Decomposition (AD)[1]-[3]. Targets or scatter-points in the scene will be imaged progressively in a sequence of their energy due to the greedy natural of matching pursuit employed during AD. With the novel technique, the contents in the final SAR image can be controlled by an energy threshold, besides the interested targets can be separated or extracted from the background clutter and this may be helpful to the SAR image understanding.
\end{abstract}

Index Terms - SAR imaging algorithm, Atomic decomposition, progressive imaging technique

\section{INTRODUCTION}

Synthetic Aperture Radar (SAR) is an important remote sensing method and has been widely used in various domains. Focusing of the raw data from SAR is a key step for any SAR based application. A lot of SAR imaging algorithms have been proposed [4]-[12] since the first SAR sensor was built. Most of these algorithms are designed to process the raw data indiscriminatively and pay the same attention to the clutters as well as the targets interested. These algorithms may have a good efficiency in processing time but the final SAR image may be hard to be understood due to the interference from the background clutters.

The progressive SAR imaging technique proposed in this paper introduces a new scheme to focus the SAR data. The focusing process is carried out progressively along with parameters-estimation. The SAR image is formed pixel by pixel or in groups of pixels according to the energy of scattering-point in the scene. The energy of the focused pixels is subtracted from that of the raw data and the imaging procedure will be stopped when the remaining energy is small enough or smaller than a pre-set threshold.

The progressive imaging technique pays more attention to the targets that may be concerned or have a larger energy than the clusters around them. Besides, the novel technique can extract the targets from the background round them and make them ready for analysis in latter processing steps.

One implementation algorithm of the progressive imaging technique is discussed detailed in this paper. The algorithm utilizes $\mathrm{AD}$ in the azimuth to detect the time-frequency parameters including modulation rate, time 
center, Doppler centroid and duration. With those parameters, the range migration of a scattering point can be estimated and corrected; the point can also be extracted from raw data and reconstructed in the final image. Due to the greedy nature of matching pursuit employed by $\mathrm{AD}$, the scattering points are detected and focused one by one according to their energy.

\section{PROCEDURE OF PROGRESIVE SAR IMAGING}

The processing procedure of the progressive SAR imaging technique can be summarized as follows:

(1) Focusing the raw data in the range direction via matched filter;

(2) Projecting the range-focused SAR data onto azimuth direction;

(3) Analyzing the azimuth signal via $\mathrm{AD}$ and finding the maximum-energy chirp signal with its four parameters: duration, chirp rate, time center and Doppler centriod;

(4) Estimating range migration from the four chirp parameters and reconstructing the scattering point according to those chirp signals at the location that it should be;

(5) Subtracting the estimated signal from the azimuth projected signal and repeating the same operations from step (3) to (5) until the energy of the remains is smaller than a pre-setting threshold.

From the steps listed above, the scattering points in a scene can be extracted, focused and reconstructed in a sequence determined by the amount of energy. A carefully selected threshold will make the final image much clear and free of clutter at some degree. The flow chart of the process stated above is illustrated in figure 1.

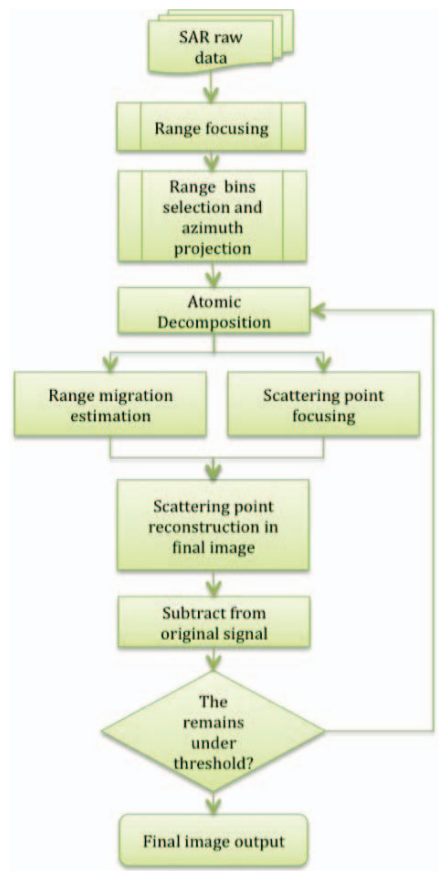

Figure 1 Flow chart of progressive SAR imaging technique 
The procedure illustrated in figure 1 can be viewed as a typical implementation of the progressive SAR imaging. The implementation is based on energy selection and assumes that the potential targets may have a higher energy level than the clutter around them. Some other potential targets judgment methods or rules can also be adopted to form new kind of implementation of the technique.

In figure 2, a comparison of the imaging results from traditional imaging algorithm and the novel progressive imaging technique is presented.
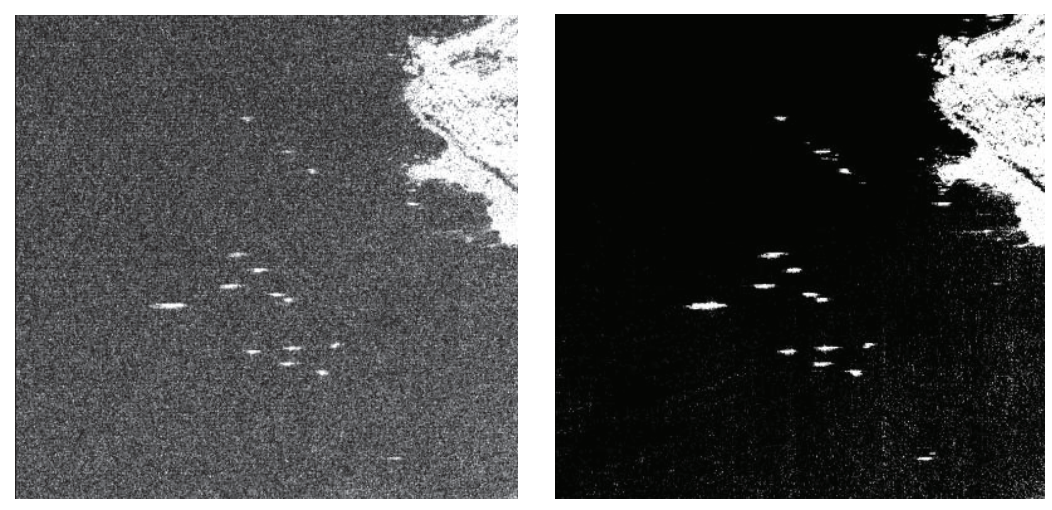

Figure 2 Comparison of the imaging results from classic algorithm and the progressive imaging technique. The left image is result of classic algorithm and the right one is from the novel technique.

From the images in figure2, it can be seen that the clutter is much reduced and the image is very clear from the result of novel technique.

\section{CONSIDERATIONS OF PROCESSING EFFICIENCY}

It is evident that the progressive SAR imaging technique may have a lower efficiency compared with traditional SAR imaging algorithms, while some methods can be adopted to improve the processing efficiency.

(1) A region selection method can be employed to search and find regions with high energy level from range focused SAR data. These regions may be appointed with a higher priority in latter progressive processing.

(2) The range of chirp rate, duration, time center and Doppler centriod of the azimuth-projected signal can be obtained before $\mathrm{AD}$ and this may help to improve the processing efficiency.

(3) Some fast algorithms of matching pursuit and chirp estimation can be employed to enhance the processing efficiency.

\section{APPLICATIONS OF PROGRESIVE SAR IMAGING}

The novel imaging technique proposed in this paper can be regarded as a kind of smart imaging algorithms. The progressive SAR imaging technique pays more attention to the scattering points that may be part of a target. Thus, such a technique can be used as a targets detector at signal level. The technique can yield not only the image of 
scattering points in the scene but also the parameters of those points. From these parameters, the moving feature can be estimated and points can also be selected and grouped to form a target objet. Besides, the technique can achieve a fine focusing performance to each scattering point for a special set of focusing parameters are used to each point according to the result from $\mathrm{AD}$. The affection from range migration is very little due to the azimuth projection operation.

\section{REFERENCES}

[1] OMAR A. YESTE-OJEDA, JESUS GRAJAL, and GUSTAVO LÓPEZ-RISUENO, “Atomic Decomposition for Radar Applications”, IEEE TRANSACTIONS ON AEROSPACE AND ELECTRONIC SYSTEMS, VOL. 44, NO. 1, JANUARY 2008

[2] López-Risueño G., and Grajal J, "Multiple signal detection and estimation using atomic decomposition and EM". IEEE Transactions on Aerospace and Electronic Systems, 42 84-102, Jan. 2006

[3] Paul Leducq, Laurent Ferro-Famil, and Eric Pottier, "Matching-Pursuit-Based Analysis of Moving Objects in Polarimetric SAR Images”, IEEE GEOSCIENCE AND REMOTE SENSING LETTERS, VOL. 5, NO. 2, pp123-127, APRIL 2008

[4] C. Cafforio, C. Prati and F. Rocca. SAR data focusing using seismic migration techniques IEEE Trans on AES, Vol27 No.2 1991

[5] Richard Bamler. A Comparison of Range-Doppler and Wavenuber Domain SAR Focusing Algorithms. IEEE Trans on GRS, Vol30 No4 July 1992

[6] R. Keith Raney. Precision SAR Processing Using Chirp Scaling. IEEE Trans on Geoscience and Remote Sensing, Vol.32, No.4, July, 1994.

[7] Knut Eldhuset, “A New Fourth-Order Processing Algorithm for Spaceborne SAR” IEEE Trans on AES, Vol.43, NO.3 July, 1998.

[8] Knut Eldhuset, "Ultra high resolution spaceborne SAR processing", IEEE Transactions on Aerospace and Electronic Systems, vol.40, No.1, Jan 2004 page:370 - 378.

[9] Alberto Moreira, Josef Mittermayer and Rolf Scheiber. Extended Chirp Scaling Algorithm for Air- and Spaceborne SAR Data Processing in Strimpmap and ScanSAR Imaging Modes. IEEE Trans on Geoscience and Remote Sensing, vol.43, No.5, Sep, 1996.

[10] Moreira, A.; Yonghong Huang. Airborne SAR processing of highly squinted data using a chirp scaling approach with integrated motion compensation. IEEE Transactions on Geoscience and Remote Sensing, vol. 32, No. 5, Sept. 1994 page:1029 - 1040

[11] Frank H. Wong and Tat Soon Yeo, "New Applications of Nonlinear Chirp Scaling in SAR Data Processing", IEEE Trans on Geoscience and Remote Sensing, vol. 39, No. 5, May 2001

[12] Davidson, G.W., Cumming, I.G. and Ito, M.R., "A Chirp-Scaling Approach for Processing Squint Mode SAR Data", IEEE Trans on Aerospace and Electronic Systems, vol.32, No.1, Jan. 1996, pages:121 - 133 